\title{
Psychothérapie interpersonnelle et des rythmes sociaux (PTIRS) dans le trouble bipolaire II : structure du traitement et exemples cliniques

\author{
IPSRT for Bipolar II Disorder: Treatment development and case \\ examples \\ Psicoterapia interpersonal y de ritmos sociales (PTIRS) en el \\ trastorno bipolar II: Estructura del tratamiento y ejemplos clínicos \\ Psicoterapia interpessoal e de ritmos sociais (TIPRS) no transtorno \\ bipolar II: Estrutura do tratamento e exemplos clínicos
}

\author{
Holly A. Swartz, Ellen Frank et Debra Frankel
}

Volume 33, numéro 2, automne 2008

La psychothérapie interpersonnelle

URI : https://id.erudit.org/iderudit/019673ar

DOI : https://doi.org/10.7202/019673ar

Aller au sommaire du numéro

Éditeur(s)

Revue Santé mentale au Québec

ISSN

0383-6320 (imprimé)

1708-3923 (numérique)

Découvrir la revue

Citer cet article

Swartz, H. A., Frank, E. \& Frankel, D. (2008). Psychothérapie interpersonnelle et des rythmes sociaux (PTIRS) dans le trouble bipolaire II : structure du traitement et exemples cliniques. Santé mentale au Québec, 33(2), 151-184. https://doi.org/10.7202/019673ar

\section{Résumé de l'article}

Le trouble bipolaire II (BP II) est une pathologie psychiatrique fréquente, récurrente et débilitante. Pourtant, peu d'études ont évalué quelles étaient les meilleures approches pour le traitement de cette maladie. La psychothérapie interpersonnelle et des rythmes sociaux (PTIRS) [Interpersonal and Social Rythm Therapy] a démontré une utilité dans le traitement du trouble bipolaire I, en association avec la médication. Contrairement au trouble BP I, le trouble BP II est caractérisé par des épisodes atténués, non psychotiques, de manie (hypomanie), de sorte qu'il ne semble pas y avoir de contre-indications à l'emploi de la PTIRS en monothérapie. Cette approche combine une approche comportementale visant à augmenter la régularité des routines quotidiennes, avec une psychothérapie interpersonnelle qui aide les patients à mieux gérer les multiples problèmes psychosociaux associés à cette pathologie chronique. Une description est faite des conceptions théoriques sous-tendant l'utilisation de la PTIRS dans le trouble bipolaire. Une brève description du trouble BP II est faite. Plusieurs modifications sont apparues nécessaires, dans notre expérience, pour adapter la PTIRS au traitement du trouble BP II (en comparaison avec le trouble BP I), en raison des caractéristiques cliniques particulières de cette population, notamment l'instabilité du tableau clinique, la difficulté à repérer le type d'épisode en cours parce que les symptômes sont souvent mixtes (intrication entre symptômes d'activation et de dépression), et également en raison du chevauchement symptomatique ou de la comorbidité avec le trouble de personnalité borderline. Il existe également une comorbidité fréquente avec le trouble d'abus ou de dépendance aux substances psychoactives. Des vignettes tirées de notre expérience clinique tentent d'illustrer diverses problématiques courantes rencontrées dans la thérapie de cette population, et qui sont reliées aux caractéristiques ci-haut mentionnées du trouble BP II. La PTIRS apparaît être un ajout important et intéressant aux approches de traitement du trouble BP II parce qu'elle tient compte des divers aspects de cette pathologie ; cette approche nous est apparue efficace dans cette étude préliminaire et nous sommes d'avis que des études systématiques ultérieures sont requises et permettront d'évaluer de façon plus formelle son efficacité dans le traitement du trouble bipolaire II. 


\title{
Psychothérapie interpersonnelle et des rythmes sociaux (PTIRS) dans le trouble bipolaire II : structure du traitement et exemples cliniques
}

\author{
Holly A. Swartz* \\ Ellen Frank** \\ Debra Frankel***
}

Le trouble bipolaire II (BP II) est une pathologie psychiatrique fréquente, récurrente et débilitante. Pourtant, peu d'études ont évalué quelles étaient les meilleures approches pour le traitement de cette maladie. La psychothérapie interpersonnelle et des rythmes sociaux (PTIRS) [Interpersonal and Social Rythm Therapy] a démontré une utilité dans le traitement du trouble bipolaire I, en association avec la médication. Contrairement au trouble BP I, le trouble BP II est caractérisé par des épisodes atténués, non psychotiques, de manie (hypomanie), de sorte qu'il ne semble pas y avoir de contre-indications à l'emploi de la PTIRS en monothérapie. Cette approche combine une approche comportementale visant à augmenter la régularité des routines quotidiennes, avec une psychothérapie interpersonnelle qui aide les patients à mieux gérer les multiples problèmes psychosociaux associés à cette pathologie chronique. Une description est faite des conceptions théoriques sous-tendant l'utilisation de la PTIRS dans le trouble bipolaire. Une brève description du trouble BP II est faite. Plusieurs modifications sont apparues nécessaires, dans notre expérience, pour adapter la PTIRS au traitement du trouble BP II (en comparaison avec le trouble BP I), en raison des caractéristiques cliniques particulières de cette population, notamment l'instabilité du tableau clinique, la difficulté à repérer le type d'épisode en cours parce que les symptômes sont souvent mixtes (intrication entre symptômes d'activation et de dépression), et également en raison du chevauchement symptomatique ou de la comorbidité avec le trouble de personnalité borderline. Il existe également une comorbidité fréquente avec le trouble d'abus ou de dépendance aux substances psychoactives. Des vignettes tirées de notre expérience clinique tentent d'illustrer diverses problématiques courantes rencontrées dans la thérapie de cette population, et qui sont reliées aux caractéristiques ci-haut mentionnées du trouble BP II. La PTIRS apparaît être un ajout important et intéressant aux approches de traitement du trouble BP II parce qu'elle tient compte des divers aspects de cette pathologie; cette approche nous est apparue efficace dans cette étude préliminaire et nous sommes d'avis que des études systématiques ultérieures sont requises et permettront d'évaluer de façon plus formelle son efficacité dans le traitement du trouble bipolaire II.

\footnotetext{
* M.D., Department of Psychiatry, Western Psychiatric Institute and Clinic, University of Pittsburgh, Pittsburgh, Pennsylvania.

** Ph.D., Department of Psychiatry, University of Pittsburgh.

*** L.C.S.W., Department of Psychiatry, University of Pittsburgh.

\section{Remerciements}

Ce travail a reçu des subventions du National Institute of Mental Health 64518 and 30915 et du NARSAD Young Investigator Award ( $\mathrm{D}^{\mathrm{r}}$ Swartz).

Traduit de l'anglais par J. Leblanc, m.d. et U. Streit, Ph.D., Montréal, Québec. 
e trouble bipolaire II (BP II) est une pathologie psychiatrique courante, récurrente et débilitante. Les individus qui en souffrent forment une partie importante de la clientèle des services externes de psychiatrie, mais des approches thérapeutiques pertinentes et efficaces sont encore peu développées. La psychothérapie représente une option potentiellement importante pour ces sujets mais elle n'a pas encore fait l'objet d'études adéquates. Le trouble BP II est caractérisé par des épisodes atténués et non psychotiques d'accélération maniaque (épisodes hypomaniaques), et il n'y a pas de contre-indication nette à l'utilisation de psychothérapie en monothérapie dans ce syndrome (alors que le traitement de l'épisode maniaque franc du trouble BP I implique d'office une médication). La psychothérapie a l'avantage sur la pharmacothérapie d'aider les patients à mieux gérer les fréquentes problématiques psychosociales associées à cette maladie chronique. Une psychothérapie efficace du trouble BP II représenterait une option intéressante pour les patients, notamment ceux qui hésitent à utiliser une médication associée à des risques potentiels ou à des effets secondaires gênants. Il n'existe cependant pas, dans la littérature scientifique, de données probantes pour appuyer l'utilisation d'une psychothérapie dans le trouble BP II.

La psychothérapie interpersonnelle et des rythmes sociaux (PTIRS) combine une approche comportementale visant à augmenter la régularité des routines quotidiennes, avec une psychothérapie interpersonnelle (PTI); ce traitement s'est avéré efficace comme psychothérapie du trouble BP I en association avec une médication (Frank, 2005). Son efficacité a également été démontrée dans le traitement de l'épisode de dépression bipolaire du trouble BP I ou II en association avec une pharmacothérapie (Miklowitz et al., 2007). Elle n'a pas, cependant, été testée à date comme traitement du trouble BP II en l'absence de pharmacothérapie. Cet article décrit brièvement le trouble bipolaire II et la PTIRS, de même que le processus d'adaptation de la PTIRS pour le traitement de cette pathologie. Il présente ensuite une série de vignettes cliniques choisies à partir de notre expérience avec la PTIRS utilisée en monothérapie dans le traitement de l'épisode de dépression chez le bipolaire II.

La PTIRS possède une nette validité comme traitement de cette population et nos expériences avec cette approche ont été à ce jour, positives. Nous estimons toutefois, que la PTIRS requiert d'autres études systématiques afin d'évaluer de façon plus formelle son efficacité comme traitement du trouble bipolaire II. 


\section{Description du trouble bipolaire II}

Le trouble bipolaire II a d'abord été décrit comme entité clinique distincte dans les années 1970 (Dunner et al., 1976) et a été inclus dans la nomenclature officielle du DSM dans la version de 1994. Le DSM-IV définit le trouble BP II comme un sous-type du trouble bipolaire, caractérisé par la présence actuelle ou passée d'au moins un épisode de dépression majeure et d'un épisode hypomaniaque (American Psychiatric Association, 1994). La présentation la plus typique est l'existence de plusieurs épisodes de dépression majeure, et des hypomanies en moins grand nombre, avec présence d'au moins certains symptômes environ $50 \%$ du temps (Judd et al., 2003). Les premières descriptions suggéraient que le trouble BP II était une forme plus bénigne du trouble BP I parce que, par définition, les individus qui en souffraient ne présentaient pas de manie franche. Avec le temps, cependant, il appert que le trouble BP II, caractérisé par la présence d'épisodes multiples et prolongés de dépression, est au moins autant, sinon plus, débilitant que le trouble BP I.

Le trouble BP II est fréquent. La prévalence à vie se situe entre 0,5\% (Regier et al., 1993) et 5,3\% (Angst, 1998) ou même $11 \%$ (Angst et al., 2003). Si l'on assume que la prévalence réelle se situe quelque part vers le milieu de ces estimés, on peut en conclure que le trouble bipolaire II affecte de 2 à 3 fois plus d'individus que le trouble bipolaire I.

L'expérience cumulative est à l'effet que le trouble BP II, tout comme le trouble BP I, est une maladie chronique et que des symptômes persistent longtemps au delà de la «rémission» des épisodes syndromiques. Judd et ses collègues (2003) ont comparé l'évolution sur dix ans de 206 sujets rencontrant les critères RDC (Research Diagnostic Criteria) du trouble BP I $(\mathrm{n}=135)$ ou du trouble BP II $(\mathrm{n}=71)$ dans le cadre de la NIMH Collaborative Depression Study. À l'entrée dans l'étude les sujets BP I étaient plus sévèrement atteints (risque plus grand d'hospitalisation, scores plus faibles à l'échelle GAS d'évaluation globale, et prévalence accrue de symptômes psychotiques) mais les sujets BP II ont présenté une évolution longitudinale plus chronique, ils étaient moins susceptibles de retrouver leur niveau de fonctionnement prémorbide entre les épisodes et les périodes d'euthymie étaient plus courtes. Les auteurs ont évalué cette même population sur une période de suivi de 20 ans, et ils ont décrit que les symptômes dépressifs prévalaient sur les symptômes hypomaniaques/maniaques chez les sujets BP I et chez les BP II, mais le rapport était 10 fois plus grand chez les sujets BP II : ratio de $3: 1$ pour les BP I, et $37: 1$ pour les BP II (Judd et al., 2003). Les sujets BP II sont plus susceptibles de présenter une 
cyclicité rapide que les BP I (Akiskal et al., 2000) et de présenter un pattern saisonnier de fluctuations de l'humeur (Vieta et al., 1997). Les diagnostics de BP I et de BP II apparaissent stables dans le temps (Coryell et al., 1995 ; Coryell et al., 1989), ce qui tend à confirmer l'évidence croissante à l'effet que ces deux phénotypes (BP I et BP II), malgré des chevauchements symptomatologiques, ont des caractéristiques spécifiques au niveau de leur évolution, de leurs répercussions, de leurs histoires familiales et peut-être de leur étayage génétique.

Les états mixtes sont très fréquents dans le trouble BP II et ils ont des répercussions cliniques énormes. Benazzi et ses collègues ont défini les états dépressifs mixtes (DMX) comme des «traits d'hypomanie dans un contexte d'épisode de dépression majeure (EDM)» (Benazzi, 2001a; Benazzi, 2001b ; Benazzi, 2001d). Ces épisodes peuvent différer de la définition du DSM-IV pour les états mixtes en ce qu'il n'est pas nécessaire de retrouver chez un individu tous les critères d'un épisode hypomaniaque lors d'un épisode de dépression majeure. Ces auteurs définissent comme DMX3 un épisode de dépression majeure durant lequel au moins trois symptômes ou signes d'hypomanie sont présents à l'intérieur de l'épisode. Dans un échantillon de 97 patients porteurs de dépression BP II n'ayant pas reçu de médication, la DMX3 se retrouvait chez $42 \%$ de l'échantillon (73\% avaient au moins 2 symptômes d'hypomanie). Ces caractéristiques étaient présentes avec une fréquence significativement plus grande chez les individus porteurs de dépression BP II que chez les déprimés unipolaires ( $<$ < 0,05) (Benazzi, 2001c). Chez les sujets avec dépression BP II, les symptômes hypomaniaques concomitants les plus fréquents étaient l'irritabilité, la distractibilité, la tachypsychie, et la volubilité. Ces données illustrent que plusieurs individus avec un trouble BP II présentent des symptômes d'hypomanie atténués même quand ils sont déprimés. De fait, Benazzi et al. ont proposé que la présence de symptômes dépressifs atypiques, et la cooccurrence de DMX3 peuvent être un bon marqueur transversal permettant de distinguer la dépression BP II de la dépression unipolaire (puissance de prédiction $=0,75)($ Benazzi, 2001b). Vus sous l'angle de la clinique, les états mixtes sont vécus comme des périodes de dysphorie, d'inconfort et de survoltage, qu'un patient avait décrit comme être épuisé et "sur-les-nerfs». La complexité de ce regroupement symptomatique rend difficile l'observation de variations de l'humeur. Dans le cas du trouble BP II, la conceptualisation classique de cette maladie comme étant caractérisée par des épisodes successifs avec des polarités distinctes et opposées n'est pas exacte, et porte même à confusion pour les patients et pour leurs thérapeutes. 


\section{Description de la PTIRS}

La psychothérapie interpersonnelle et des rythmes sociaux (PTIRS) est une forme de traitement combinant une approche comportementale visant à augmenter la régularité des routines quotidiennes, et une psychothérapie interpersonnelle (PTI) adaptée aux besoins des patients porteurs d'un trouble PB I; elle a été développée comme psychothérapie associée à la médication (Frank et al., 2000). Le cadre conceptuel de la PTIRS repose sur trois hypothèses inter reliées : 1) le «modèle d'instabilité » du trouble bipolaire mis de l'avant par Goodwin et Jamison (1990), 2) les théories sur l'impact des stimuli environnementaux et sociaux dans la facilitation et/ou le dérèglement de l'intégrité du rythme circadien (Ehlers et al., 1988; Ehlers et al., 1993), 3) les principes de la PTI dans le traitement de la dépression unipolaire. La PTIRS intègre des stratégies psychoéducationnelles, interpersonnelles et comportementales dans le but de réduire les symptômes, d'améliorer le fonctionnement global et de prévenir la récurrence d'épisodes.

Dans leur modèle d'instabilité, Goodwin et Jamison décrivent trois voies interreliées vers la récurrence d'un épisode BP I: des événements de vie éprouvants, la non observance à la médication, et la disruption des rythmes circadiens (Goodwin et Jamison, 1990). Chacune des voies conduisent un individu bipolaire stable vers un épisode de dépression ou de manie. Leur modèle suggère que les individus porteurs d'un trouble bipolaire sont fondamentalement (biologiquement) vulnérables aux disruptions des rythmes circadiens. Les stresseurs psychosociaux interagissent avec la vulnérabilité biologique dans la production de symptômes. À titre d'exemple, un événement de vie stressant comme la naissance d'un enfant modifie les rythmes sociaux, ce qui entraîne une perturbation de l'intégrité circadienne, qui peut provoquer une récurrence. De même, des relations interpersonnelles problématiques, ou des cédules irrégulières de travail, peuvent modifier les signaux régulateurs sociaux quotidiens et engendrer ainsi une instabilité des rythmes sociaux. Une conséquence directe de ce modèle se retrouve dans l'hypothèse que l'apprentissage par les patients d'une vie plus ordonnée et de la résolution plus efficace de leurs problèmes interpersonnels devrait entraîner une intégrité circadienne et ainsi diminuer le risque de récurrence.

Les recherches sur les rythmes circadiens ont permis d'identifier des relations réciproques entre les rythmes circadiens, les cycles veillesommeil et l'humeur. On a bien documenté le lien entre une réduction du sommeil et la survenue de manie chez les sujets bipolaires (Leibenluft et al., 1996; Wehr et al., 1987). De plus, la privation de 
sommeil a des effets antidépresseurs significatifs (bien que transitoires) chez les sujets unipolaires et bipolaires (Barbini et al., 1998; Leibenluft et al., 1993; Leibenluft et Suppes, 1999). Ehlers et al. (1988) ont tenté de relier les modèles biologiques et psychosociaux de la dépression, et formulé l'hypothèse que des signaux sociaux spécifiques mobilisent les rythmes circadiens (Zeitgeber) et d'autres vont plutôt les perturber (Zeitstörer). On peut définir les Zeitgeber comme des relations personnelles, des demandes sociales ou des tâches qui mobilisent les rythmes biologiques (par exemple, se lever à $7 \mathrm{~h}$ chaque matin pour conduire les enfants à l'école). Dans cette perspective, la perte d'un Zeitgeber social (par exemple, les vacances d'été qui ne requièrent plus une telle régularité de lever pour préparer l'enfant pour l'école) pourrait déclencher un épisode en raison de la dysrégulation de rythmes biologiques (Ehlers et al., 1993). Un travail régulier est un autre exemple de Zeitgeber social. La perte d'un travail qui déterminait les horaires du lever et du coucher, des périodes de repos et l'heure des repas, est un Zeitgeber perdu. Chez un individu avec une prédisposition génétique à la dépression, les perturbations physiologiques et chronobiologiques engendrées par la perte de signaux régulateurs de l'heure des repas et du sommeil pourraient être aussi importantes dans la genèse d'un épisode que la détresse psychologique générée par l'événement.

Par contraste avec les Zeitgeber, les Zeitstörer sont définis comme des événements d'origine physique, chimique ou psychosociale, qui perturbent l'horloge biologique. Par exemple, voyager à travers des fuseaux horaires représente un prototype de Zeitstörer. Les changements abrupts dans les cédules d'exposition à la lumière, des temps de repos et du sommeil, peuvent produire une gamme de symptômes allant du simple inconfort lié au décalage horaire à un épisode affectif net chez un individu prédisposé. D'autres exemples de Zeitstörer potentiels incluent la naissance d'un bébé, les séparations de couple, le travail sur appel chez les médecins, les dates de tombée pour un travail (qui peuvent amener à travailler tard la nuit et sauter repas et heures de sommeil), et le travail posté. Chaque perturbation peut altérer les rythmes circadiens et les cycles veille-sommeil, et éventuellement déclencher un épisode affectif. La PTISR est fondée sur la prémisse qu'aider les patients à régulariser les rythmes sociaux (moduler les Zeitgeber et les Zeitstörer) peut aider à réduire, chez les sujets prédisposés, les risques de développement de symptômes dépressifs et (hypo) maniaques.

La PTISR est formée de trois composantes: la psychoéducation, les interventions sur les rythmes sociaux et les interventions 
interpersonnelles. Dans la pratique clinique, ces stratégies sont appliquées de façon fluide et flexible, sans frontières distinctes entre les diverses modalités. Au cours d'une séance, le thérapeute va d'une technique à l'autre en fonction des besoins des patients. Voici un aperçu de ces diverses composantes.

La psychoéducation met l'accent sur a) la maladie et ses conséquences, b) les options de traitement et les effets secondaires qui en découlent (y compris la médication), et c) la détection de symptômes prodromiques ou de signes précoces de rechute. Les thérapeutes recueillent des informations auprès des patients sur leurs symptômes et l'évolution de leur trouble bipolaire, l'impact de la maladie sur leur fonctionnement social et professionnel, et sur les médications qu'ils ont utilisées (le cas échéant) pour traiter leur maladie. Les thérapeutes donnent un nom à la pathologie, amorçant ainsi le processus de psychoéducation. Au cours du déroulement de la PTISR, les patients sont invités à devenir des «experts» du trouble bipolaire de façon à pouvoir collaborer plus efficacement dans la prise en charge de leur maladie. Dans le but de faciliter l'identification précoce de symptômes prodromiques, le thérapeute passe en revue avec le patient les épisodes antérieurs de dépression et de manie. Ensemble ils identifient les comportements ou symptômes caractéristiques du début d'un épisode et ils s'entendent pour être à l'affût d'une façon routinière de ces signes avant-coureurs, chez le patient, d'une exacerbation possible.

La thérapie des rythmes sociaux (TRS) est fondée sur la théorie que les rythmes quotidiens stables engendrent une plus grande stabilité de l'humeur. Cette composante du traitement vise le développement de stratégies pour établir des Zeitgeber sociaux réguliers et stabilisateurs des rythmes biologiques, et la protection contre l'impact négatif des zeitstörer déstabilisants. Les patients remplissent chaque semaine un instrument appelé Mesure des rythmes sociaux (MRS - en anglais, le Social Rhythm Metric, ou SRM) qui aide à optimiser la stabilité de leurs rythmes quotidiens. Les premières 3 ou 4 semaines de MRS servent à définir la ligne de base de leurs rythmes sociaux. Patients et thérapeutes revoient ensemble les MRS et identifient les portions de stabilité et d'instabilité de ces rythmes. Par exemple, si l'heure du coucher est adéquate durant la semaine, l'est-elle également au cours du week-end? L'humeur du patient plonge-t-elle les jours où il saute des repas ou n'a pas de contacts avec d'autres personnes? L'examen des feuillets aide à identifier des comportements qui ont un impact négatif sur la stabilité des rythmes. Lorsque la ligne de base est documentée et que les patterns de régularité/irrégularité sont identifiés, thérapeutes et patients axent 
leurs efforts vers la stabilité des rythmes par des étapes de changements graduels de styles de vie, à travers des objectifs à court, moyen et long terme, de façon à réduire les fluctuations de ces rythmes. À titre d'exemple, un objectif court terme pourrait être de se mettre au lit à heure fixe pendant une semaine. Pour atteindre cet objectif, le patient peut devoir changer certains comportements sociaux (par exemple, réduire les soirées sociales de fin de journée), ou activités sportives (par exemple, effectuer les exercices physiques le matin plutôt que le soir). Le thérapeute peut aider le patient à trouver un temps de lever plus adéquat le matin, en modifiant progressivement l'heure actuelle du lever. Des buts intermédiaires pourraient être d'arriver à dormir 8 heures par nuit et ne pas faire de sieste le jour, ou adopter une activité quotidienne régulière, comme un travail à temps partiel ou des activités de bénévolat. Pour atteindre ces objectifs, les patients viseront des gains à court terme mais pourront aussi aménager leurs activités (par exemple, s'inscrire à des cours en après-midi pour réduire le besoin de sieste). Les thérapeutes insistent sur l'importance d'une cédule de vie régulière, même si cette cédule doit être décalée pour mieux rencontrer les tendances de vie du patient. Par exemple, des patients bipolaires vont parfois préférer se faire une routine de vie qui comporte un coucher tardif (par exemple, à $2 \mathrm{~h}$ de la nuit) et un lever également tardif (par exemple, à $10 \mathrm{~h}$ ). Le thérapeute aide le patient à réaliser que toute cédule régulière est acceptable dans la mesure où elle permet au patient de rencontrer ses obligations sociales, et de pouvoir obtenir des périodes de sommeil de durée continue adéquate (en général de 7 à 9 heures). Les objectifs à long terme pourraient être d'encourager les patients à dénicher des emplois qui permettent d'adhérer à de telles cédules (par exemple, un travail dans une librairie qui ne commencerait qu'à midi).

La psychothérapie interpersonnelle est une composante importante de la PTIRS. Comme la PTI, la PTIRS se déroule en trois phases (initiale, intermédiaire et phase de terminaison). Dans la même logique que le modèle d'origine, la psychoéducation et l'attribution du rôle de malade permettent au patient de reconnaître l'importance de leur maladie (ici, le trouble BP II) dans la genèse et le maintien de leurs problèmes interpersonnels. Tout comme dans le modèle original, le thérapeute doit mettre l'accent et identifier une aire problématique interpersonnelle. Par ailleurs la PTIRS se distingue de la PTI sous divers aspects. En premier lieu, la PTI met l'accent sur les liens entre les événements de vie et l'humeur, alors que dans la PTIRS les événements de vie sont considérés à la fois comme des sources de dysrégulation de l'humeur et comme des disrupteurs potentiels des rythmes sociaux. La PTIRS aborde donc les problèmes interpersonnels avec les stratégies 
habituelles de la PTI, et aussi avec des stratégies comportementales visant à régulariser les perturbations des rythmes sociaux associés aux problèmes interpersonnels. La PTIRS a, de plus, ajouté un cinquième focus (ou aire problématique), le deuil de l'intégrité du soi (grieving for the lost healthy self); l'accent mis sur ce focus permet aux patients de faire le deuil de l'image qu'ils se font de la personne qu'ils auraient pu être, n'eut été de la maladie bipolaire, et d'accepter les limites que la maladie apporte à leur style de vie et à leur fonctionnement optimal.

\section{Adaptation de la PTIRS pour le traitement du trouble BP II}

Au point de départ, nous avions émis l'hypothèse que la PTIRS, même utilisée en monothérapie, représentait un traitement prometteur du trouble BP II parce que: a) la composante PTI (psychothérapie interpersonnelle) de la PTIRS est un outil efficace pour le traitement des symptômes dépressifs, et les phases dépressives prédominent dans le trouble BP II ; b) la composante TRS (thérapie des rythmes sociaux) de la PTIRS permet de contrer l'hypomanie et la composante hypomaniaque des symptômes mixtes souvent présent dans la dépression BP II ; c) la PTIRS permet un «dosage» flexible des composantes PTI et TRS en fonction de la présentation clinique - très variable dans le trouble BP II ; d) la PTIRS possède un ensemble significatif de stratégies pouvant aider le patient à faire face aux déficits fonctionnels chroniques résultant de cette maladie. Par ailleurs, les besoins thérapeutiques des patients souffrant de dépression BP II diffèrent de ceux des patients avec trouble BP I; nous avons donc apporté une série d'ajustements et d'adaptation de façon à rendre cette approche plus appropriée pour cette population. Nous décrivons ci-après les problématiques spécifiques apparues au cours du traitement des patients souffrant de trouble BP II par la PTIRS et les stratégies utilisées pour y remédier.

\section{Explication de l'importance de la régularité des rythmes sociaux}

Pour atteindre une régularité des rythmes sociaux, tel que proposé dans la PTIRS, les patients doivent apporter des changements importants dans leurs cédules quotidiennes, ce à quoi ils sont souvent réticents. Avec les patients $\mathrm{BP} \mathrm{I}$, les thérapeutes mettent régulièrement de l'avant les aspects destructeurs des épisodes maniaques antérieurs, de façon à les inciter à effectuer les changements nécessaires dans leurs routines de vie. Avec les sujets avec un trouble BP II, il est moins évident pour le patient, du moins au début, d'effectuer des changements en apparence non nécessaires (par exemple, aller au lit à heures régulières chaque nuit). L'explication à l'effet que ces changements vont aider à prévenir une hypomanie ne convainc pas d'emblée plusieurs de ces 
individus. Au contraire, certains patients voient l'hypomanie comme un soulagement à la suite de leurs dépressions récurrentes, et ne souhaitent pas vraiment en prévenir la survenue. Le raisonnement appuyant la nécessité de changements dans les rythmes sociaux, approprié avec les sujets BP I, n'est donc pas nécessairement applicable avec les patients BP II. De surcroit, les patients avec trouble BP II ont moins d'insight par rapport à leur maladie que les patients avec trouble BP I (Pallanti et al., 1999) et souvent ne reconnaissent même pas qu'ils ont présenté des symptômes hypomaniaques - et, encore moins, que ces symptômes compliquent le cours de leur maladie et contribuent à une évolution défavorable. Dans notre expérience, l'argumentation la plus porteuse en faveur de la régularité des rythmes sociaux, pour les patients avec trouble BP II, est celle qui met de l'avant la probabilité qu'un épisode dépressif fera suite à une hypomanie. Nous disons à nos patients à peu près ceci: "Une cédule de vie plus régulière aide à stabiliser votre humeur. Je sais que la dernière chose que vous souhaitez est de vous défaire de vos épisodes de high. Vous avez bien fait valoir que ces périodes vous sont très utiles dans votre vie professionnelle et dans votre vie personnelle. Le problème vient cependant surtout du fait que de pénibles dépressions font régulièrement suite à vos périodes d'accélération. Nous sommes, vous et moi, d'accord que les dépressions sont très débilitantes. Notre but ici est, en conséquence, de prévenir les dépressions en amenant chez vous une plus grande stabilité de l'humeur. On peut même arriver à ce que vous gardiez un peu des aspects positifs de ces périodes de high, mais il faut penser à réduire leur intensité pour pouvoir prévenir les dépressions subséquentes. »

Un sous-groupe de patients BP II identifie facilement les aspects problématiques de leurs hypomanies, en particulier ceux qui présentent de l'irritabilité, de l'agitation, une réactivité de l'humeur et une distractibilité au cours de leurs périodes d'exaltation. Ces individus peuvent souhaiter éviter l'hypomanie dès le début du traitement. Pour d'autres, les aspects destructeurs de l'hypomanie (par exemple, impact négatif sur les relations interpersonnelles, dépenses impulsives exagérées, jugement déficitaire), ne deviennent apparents qu'en cours de traitement, et c'est à ce moment que le thérapeute peut intervenir plus efficacement pour prévenir l'hypomanie par une meilleure régularisation des rythmes. En début de thérapie, cependant, le levier le plus utile au thérapeute est l'argument que la prévention de l'hypomanie est susceptible de prévenir une dépression subséquente.

Nous avons constaté que certains patients vont d'abord se conformer à la consigne de changements des rythmes sociaux, mais avec une 
bonne dose de scepticisme, c'est-à-dire, qu'ils n'adhérent pas au modèle avec conviction. De façon paradoxale, ces sujets semblent tirer profit d'une détérioration transitoire de leur humeur (en mode dépressif ou hypomaniaque) suite à une période de stabilité, ce qui leur permet d'identifier de façon prospective le lien entre la stabilité des rythmes et l'humeur. Les patients capables d'observer leurs variations de l'humeur lorsqu'ils s'écartent d'une routine régulière sont plus susceptibles de maintenir dans le temps une stabilité des rythmes. Une psychothérapie de durée suffisante pour permettre aux patients de «tester» l'hypothèse des rythmes sociaux dans leur propre vie peut contribuer, à plus long terme, à une meilleure adhésion au modèle.

\section{Difficulté à régler le niveau de stimulation}

Un des principes de la PTIRS est que les thérapeutes doivent aider les patients à ajuster le niveau de stimulation dans leur vie quotidienne en fonction de leur état affectif. Les thérapeutes incitent les patients à élever le niveau de stimulation lorsque leur humeur s'abaisse ou durant les périodes de l'année où ils sont régulièrement vulnérables à la dépression, et à diminuer les stimulations ambiantes quand ils commencent à ressentir un début de symptômes d'hypomanie, ou durant les périodes de l'année où ils sont plus vulnérables à l'hypomanie ou à la manie. Les sujets porteurs d'un trouble BP II semblent avoir du mal à moduler le niveau de stimulation dans leur vie. Plusieurs semblent ne connaître que deux vitesses: «en marche» ou «à l'arrêt». Par exemple, un patient se voit comme pouvant être ou bien un «légume» ou bien travailler à trois endroits à la fois tout en suivant des cours du soir. Ceci peut découler d'un pattern dans lequel les périodes d'hypomanie sont employées à récupérer les déficits accumulés en période dépressive au niveau des responsabilités personnelles, ou encore de la difficulté à tolérer les états affectifs à tonalité négative. Ou encore, certains patients se fixent des objectifs irréalistes quand ils sont en hypomanie et ils se retrouvent à être incapables d'assumer ces tâches lorsque leur niveau d'élation s'estompe et qu'ils retrouvent leur état antérieur d'euthymie. En pratique, cela indique que les patients peuvent avoir à être aidés pour réaliser qu'il existe des intermédiaires entre «en marche» et «à l'arrêt», c'est-à-dire qu'un patient pourrait avoir un seul travail régulier plutôt que naviguer entre l'inertie complète et le surinvestissement. Les patients peuvent avoir besoin d'aide pour arriver à trouver le type d'activités reliées à un niveau approprié de stimulation; le thérapeute ne doit pas assumer que les patients vont d'emblée pouvoir le découvrir par eux-mêmes.

Souvent des sujets porteurs d'un trouble BP II s'engagent dans des activités hautement stimulantes lorsque leur humeur et leur niveau 
d'énergie s'abaissent. Cette stratégie correspond à une sorte de tentative ultime pour se mettre à l'abri de la dysphorie associée à la dépression. Cette approche peut certes être parfois utile, mais par contre la recherche de stimulation conduit souvent à des mauvais choix: par exemple, s'engager dans des liaisons extra-maritales, ridiculiser un collègue pour ses habitudes au travail, ou se lancer dans des entreprises ambitieuses qu'ils n'arriveront jamais à rendre à terme. Au début d'un traitement, il est important d'identifier ces patterns inappropriés pour ensuite, tel que discuté plus haut, permettre au patient de développer des méthodes plus pertinentes de modulation de leurs états affectifs.

Cette régulation des stimulations devient également un objectif thérapeutique quand les patients approchent de l'euthymie. L'expérience nous a montré que les sujets avec un trouble BP II et chez qui l'humeur a fluctué rapidement pendant des années ressentent un sentiment d'ennui, de vide ou d'apathie quand leur humeur tend à se stabiliser. L'état de «normalité » leur apparaît sans relief et ils se sentent inconfortable dans cet état nouveau et non familier. Ce malaise tend donc à les faire s'écarter de leur stabilité de rythmes récemment acquise en recherchant des nouvelles stimulations. Tout comme lorsqu'ils devenaient dépressifs, ils sont enclins à faire des choix qui, espèrent-ils (consciemment ou inconsciemment), vont soulager la tiédeur de la stabilité de l'humeur, sans réaliser qu'ils sont alors éminemment susceptibles de mettre en branle une cascade d'événements qui risquent de les entraîner dans un chaos interpersonnel. Ils peuvent, par exemple, reprendre contact avec un ancien amoureux alors qu'ils sont à ce moment dans une relation sentimentale stable et positive, ou encore accepter une tâche qui comporte des échéanciers impossibles à rencontrer. Les thérapeutes sont encouragés à mettre les patients en garde contre ce phénomène avant que ceux-ci ne posent des gestes dans ce sens. Il est également utile d'encourager les patients à identifier ces comportements dysfonctionnels qu'ils ont utilisés dans le passé pour activer leur humeur, et de les aider à réaliser que ces comportements ont contribué à déstabiliser leur humeur et aussi leurs relations interpersonnelles. Par exemple, le patient qui quitte impulsivement ses emplois quand il se sent apathique ou dégoûté sera encouragé à réfléchir aux conséquences négatives d'un tel comportement (par exemple, voir ses projets de carrière déçus) et à développer des stratégies différentes, plus productives, pour relever leur humeur (par exemple, augmenter le niveau d'activité physique ou consacrer plus de temps à des activités de socialisation avec des amis).

Par ailleurs, les patients hypomaniaques peuvent se voir attirés par des activités excitantes comme une conduite automobile excessive, ou la 
prise de risques financiers. Ces activités les attirent parce qu'elles stimulent leur humeur et leur énergie. Des patients ont comparé ces expériences à l'addiction à une substance. Le thérapeute doit aider le patient à reconnaître ce pattern et à éviter ce genre d'activités lorsque son humeur tend à s'exalter.

\section{Difficulté à identifier et catégoriser les états affectifs}

Un des objectifs principaux de la PTIRS est d'assister les patients à reconnaître les signes prémonitoires d'un nouvel épisode et d'arriver à les prévenir. Comme le trouble BP II est cliniquement complexe, il n'est pas facile pour les patients d'y arriver. Il peut être difficile pour eux de détecter un début de phase dépressive s'ils se sentent en même temps activés ou «intérieurement agités » (un état mixte), ou leurs cycles peuvent être rapides au point qu'il leur devient difficile — sinon impossible - de détecter le début ou la fin d'un épisode. Les thérapeutes ayant une expérience clinique avec ce genre de population sont plus habiles à aider les patients à reconnaître et à nommer correctement ces changements d'humeur. Les thérapeutes plus novices affinent leur habileté clinique face à cette pathologie complexe avec une super-vision étroite de la part des thérapeutes plus expérimentés dans le domaine.

En plus de l'expertise clinique, nous avons pu constater que les précisions apportées par Kokopoulos sur les troubles bipolaires sont éminemment utiles avec les patients BIP II. Kokopoulos et al. (2003) ont décrit le trouble bipolaire comme étant d'abord un trouble de l'énergie plutôt que de l'humeur. Parce que les sujets avec trouble BP II présentent souvent une discordance entre leur humeur et leur niveau d'énergie, le modèle de Kokopoulos les aide à mieux caractériser leur expérience de la maladie. On demande aux sujets de détecter quotidiennement la qualité de leur humeur et leur niveau énergétique de façon à mieux identifier l'évolution de leur pathologie. Pour faciliter le processus, nous avons ajouté un item «énergie » au MRS de façon à ce que les deux composantes (énergie et humeur) puissent être cotées séparément. Cette distinction permet aux patients de distinguer entre une dépression anergique et les états dépressifs mixtes avec activation.

Malgré les aptitudes du thérapeute et les efforts du patient, les subtilités de cette pathologie peuvent rendre difficile l'identification des états affectifs - et encore plus les déclencheurs des variations de l'humeur. Dans notre expérience ce processus se déroule par étapes où patient et thérapeute apprennent d'abord à identifier les phases de la maladie, ensuite à les anticiper, pour enfin réussir à les prévenir. Les 
phases initiales du traitement se concentrent donc beaucoup plus sur l'identification des symptômes et la qualification des états affectifs que ce n'est le cas dans le trouble BP I.

\section{Problèmes interpersonnels reliés à la grandiosité et à la prétention}

La grandiosité est un symptôme de manie/hypomanie, mais par ailleurs plusieurs individus avec un trouble BP II semblent en posséder à la base un haut niveau, indépendamment de leur état symptomatique. Ce phénomène peut se traduire par des attitudes de prétention ou par une indifférence narcissique pour l'impact de leurs comportements sur les autres, quel que soit leur état symptomatique. Par exemple, ces patients peuvent vouloir transgresser les normes sociales (p. ex. justifier un vol à l'étalage, ou critiquer un style de gérance deux jours après le début d'un emploi) ou formuler des demandes inappropriées à l'entourage (p. ex. demander à un parent à la santé précaire de faire un long trajet pour une rencontre sociale plutôt que d'offrir de lui rendre visite). Il est parfois difficile de décider si ceci relève de la «personnalité » ou du trouble de l'humeur, mais ces comportements ont un impact sur les relations interpersonnelles et, conséquemment, d'importantes implications thérapeutiques.

Dans notre expérience, les patients sont en général réceptifs à l'identification de ces comportements - mais seulement lorsqu'ils sont faits de manière encourageante par un thérapeute qui a déjà établi une alliance avec eux. Les patients avec un trouble BP II peuvent apparaître au début, confiant et disposés à investir dans le processus thérapeutique, mais cette attitude peut changer, compte tenu que leur narcissisme essentiel les empêche de faire entièrement confiance au thérapeute. Il faut donc parfois plusieurs mois de psychothérapie avant que la relation thérapeutique soit suffisamment solide pour permettre au thérapeute d'aborder ces questions de prétention dysfonctionnelles. Néanmoins, quand leur humeur s'est stabilisée, les patients peuvent apprendre à reconnaître et à modifier ces comportements, contribuant ainsi à améliorer leur fonctionnement interpersonnel.

Ces problèmes de prétention et de suffisance font aussi souvent surface au sein de la relation thérapeutique (par exemple, se présenter en retard aux séances, demander des heures de rendez-vous inhabituelles, être critique du thérapeute). Ces contestations de la relation thérapeutique se voient notamment en début de traitement ou lors d'exacerbation des symptômes, ce qui, à son tour, exacerbe la grandiosité toujours présente du patient. Le thérapeute doit apprendre à tolérer et éventuellement à gérer ces défis dans le contexte thérapeutique. Même si la 
PTIRS n'est pas un traitement centré sur le transfert, la détection de changements dans la relation thérapeutique peut être utile au thérapeute, pour pouvoir ensuite aider le patient à identifier chez lui ces comportements mésadaptés qui peuvent perturber les relations interpersonnelles. Nous encourageons les thérapeutes à adopter, du moins au début, une tolérance vis-à-vis les cédules de rendez-vous et les interactions des patients, dans le but de bâtir une alliance. Plus tard dans le traitement, le thérapeute visera plutôt à éveiller le patient à ces caractéristiques comportementales reliées à la maladie, et à l'aider à mieux gérer ses relations (thérapeutiques ou autres).

\section{Problèmes liés à la régulation de l'affect}

Les sujets porteurs d'un trouble BP II rencontrent souvent des difficultés à moduler leurs états affectifs. Il n'y a pas lieu de s'en surprendre, compte tenu que l'expérience de cyclicité rapide des états affectifs diminue effectivement chez le patient son «lieu de contrôle» interne de régulation de l'affect. De plus, plusieurs patients BP II ressentent de façon plus intense leurs émotions (positives et négatives). En conséquence, les patients ne savent pas toujours comment moduler ces vécus, même lorsqu'ils ne sont pas spécifiquement reliés à un épisode affectif. La première étape pour aider les patients à exercer un meilleur contrôle sur leurs émotions consiste à les renseigner sur le fait que leur maladie les a empêchés d'apprendre plusieurs des stratégies que les individus utilisent habituellement pour gérer leurs sentiments. Nous leur enseignons ensuite des stratégies concrètes de gestion des affects. Certaines techniques, même si elles ne sont pas un focus spécifique de traitement, peuvent être proposées aux patients : techniques de relaxation (par exemple, techniques de respiration profonde), de distraction (par exemple, l'écoute de musique), d'auto-apaisement (par exemple, allumer des bougies), de gestion de la colère (stratégies de temps mort), ou d'activation de leur réseau social de soutien (par exemple, rencontrer un ami au café). Les stratégies de base de la PTI aident également les patients à moduler leur humeur, à mesure qu'ils commencent à saisir le lien entre humeur et événements de vie et à développer de meilleurs outils pour gérer leur principal domaine problématique. Quand les patients traversent une période soutenue d'euthymie, ils arrivent à mieux s' «approprier» leurs humeurs et ainsi à réaliser qu'une gestion attentive des relations interpersonnelles, associée aux stratégies décrites ci-haut, leur permet une meilleure régulation de leurs affects.

Des approches similaires ont été proposées pour la gestion d'une dysrégulation affective dans le contexte d'un trouble de personnalité 
borderline (Linehan, 1993). En fait, des auteurs sont d'avis que le trouble bipolaire et le trouble de personnalité borderline forment un continuum dont les parties, en raison d'un chevauchement des symptômes, ne peuvent être distinguées (Akiskal, 2002). D'un point de vue clinique, nous sommes plutôt d'avis que, pour les patients BP II (ou chez ces individus dont le tableau clinique est davantage bipolaire que borderline), patients et thérapeutes doivent tenir compte de l'état affectif présent avant d'amorcer l'une ou l'autre de ces stratégies. De façon générale, nous encourageons les patients à choisir une activité stimulante lorsque leur humeur et leur énergie sont basses, et à éviter ce type d'activités quand leur humeur et leur niveau d'énergie sont élevés. La psychoéducation demeure une partie importante de ce processus, pour aider les patients à faire la distinction (dans la mesure où la chose est possible) entre une variation de l'humeur reliée à la polarité d'un épisode émergent, et une dysrégulation affective reliée à des événements interpersonnels vécus comme offensants, etc.

\section{Taux élevé de comorbidité avec les abus de substance}

Beaucoup d'individus souffrent à la fois de trouble BP II et de trouble d'abus de substances. Angst et al. (2006) sont d'avis que le trouble BP II explique la plus grande partie de la comorbidité entre le trouble d'abus d'alcool et la dépression majeure. Ils ont aussi noté que le début des symptômes de bipolarité précède en général de 5 ans le début de l'abus d'alcool. Les individus porteurs de trouble BP II peuvent tenter, par la consommation de ces substances, de réduire l'inconfort associé à la dépression, aux états mixtes et à l'anxiété. De plus, la désinhibition et les troubles du jugement associés à l'hypomanie peuvent conduire à des comportements impulsifs, y compris la prise excessive de substances psychoactives.

À cause des limitations connues dans l'efficacité des thérapies de type PTI pour les abus de substances (Weissman et al., 2000), nous excluons de nos études cliniques les sujets qui rencontrent les critères d'un abus ou d'une dépendance actuelles à une substance. Nous mentionnons explicitement à ces candidats que nous ne recommandons pas la PTIRS dans le traitement des addictions et nous proposons plutôt que ces sujets porteurs d'un double diagnostic prennent contact avec les ressources spécialisées dans ce domaine. Nous avons malgré tout constaté que des patients BP II désireux de participer à nos études camouflent leur abus de substance pour être acceptés dans nos groupes, ce qui nous amène à être, dans les faits, l'équipe soignante de ces patients avec double diagnostic. Par ailleurs, plusieurs patients, en plus 
de ces raisons personnelles de cacher leur abus de substance pour pouvoir entrer dans les études, évaluent mal l'ampleur de leur problème de consommation et ils camouflent cette réalité non seulement au thérapeute mais aussi à eux-mêmes. En conséquence, il est important de s'informer à ce sujet à chaque visite et d'apprendre au patient à évaluer l'impact de ces substances sur l'humeur. Nous demandons des précisions au sujet des drogues licites et illicites, de façon à bien indiquer au patient que notre intérêt porte sur l'impact des substances psychoactives sur leur humeur et leur fonctionnement plutôt que d'en faire une enquête disciplinaire. Ainsi suggérons-nous de poser des questions aussi bien sur la consommation de caféine et de nicotine que sur l'alcool, la marijuana ou autres substances. Un questionnaire ainsi répété sur l'usage de ces substances permet aux thérapeutes d'aider les patients à mieux réaliser l'association entre variations mésadaptées de l'humeur et consommation de substances, de façon à ce que ces derniers puissent arriver à réduire leur consommation.

Fréquemment négligée par les cliniciens, la caféine demeure une des substances les plus fréquemment consommée de façon abusive en pratique clinique. Chez les BP II elle est utilisée pour exalter leur énergie durant leurs périodes de creux, et pour amplifier leur humeur durant les hypomanies. Malheureusement la caféine contribue aussi à perturber le sommeil et à exacerber l'anxiété. Une attention régulière portée à cet usage de caféine, et une invitation au patient à reconnaître le lien entre son utilisation et l'instabilité conséquente de l'humeur et du sommeil peut aider le patient à gérer de façon plus adéquate cette consommation.

Il n'est pas sans intérêt de mentionner que l'intérêt objectif et dénué de préjugés que portent les thérapeutes sur la consommation de substances, et sur la relation entre l'humeur et l'emploi de ces substances, amène parfois les patients à être plus précis dans la description de leur consommation réelle. Dans le cas de la marijuana, ces admissions viennent souvent plus tardivement dans le traitement. Certains patients croient fermement que la marijuana les aide à supporter la dysphorie et l'anxiété associées à la dépression; d'autres maintiennent que cette substance les aide à ralentir leurs pensées, diminuer leur irritabilité et réduire la distractibilité associées à l'hypomanie ou aux états mixtes. Plusieurs ne reconnaissent aucun mauvais effet à la marijuana et ne voient pas pourquoi ils cesseraient leur consommation. L'assistance du thérapeute à percevoir les connexions négatives entre l'humeur et des substances apparemment inoffensives (par exemple, la caféine) peut cependant, à la longue, amener un doute chez le patient sur 
la valeur adaptative de la marijuana pour lui, et il peut en venir à en parler plus ouvertement. Nous ne recommandons pas la PTIRS comme traitement des addictions, cependant l'emploi judicieux de techniques de «limitation des dommages » (par exemple, moins est mieux que plus) peut aider les patients à diminuer leur abus de substances, même quand ils ne veulent pas participer à des approches plus conventionnelles (et plus efficaces) comme le programme des 12 étapes; nous persisterions cependant à recommander ces approches additionnelles si une consommation active de substance persistait.

\section{Problèmes cliniques communs et histoires de cas}

La partie suivante décrit des situations cliniques fréquemment rencontrées avec cette population de patients et illustre les approches PTIRS permettant de traiter cette problématique. (Dans les histoires de cas ci-dessous toute information permettant l'identification des patients a été modifiée afin de protéger leur identité)

\section{Le patient n'accepte pas ou ne reconnaît pas la sévérité des épisodes hypomaniaques.}

Tanguy, un homme célibataire de 23 ans, décrivit un épisode d'hypomanie antérieure comme une période dans laquelle il avait eu un besoin diminué de sommeil, "un peu d'énergie supplémentaire», et pendant laquelle il avait conduit "un peu trop vite". Il nia l'importance de cet épisode et fit croire au thérapeute qu'il ne s'agissait pas d'une période ayant une signification clinique importante. Plus tard, il admit que, pendant cette période, il avait conduit à $225 \mathrm{~km} / \mathrm{h}$, avait été poursuivi par la police, et avait forcé un barrage policier. Il fut arrêté, mais ne fut pas accusé grâce aux relations de sa famille avec la police.

La non-reconnaissance de la sévérité d'un épisode hypomaniaque est un signe distinctif de patients souffrant de trouble BP II. (Mentionnons que chez les patients BP II, la tendance à sous-estimer — ou à ne pas rapporter - des symptômes hypomaniaques mène au problème fréquent d'un diagnostic erroné de dépression unipolaire plutôt que bipolaire.) Dans l'approche PTIRS, le thérapeute peut utiliser deux stratégies, par exemple, la psychoéducation et «second regard». La psychoéducation aide le patient à mieux comprendre les symptômes de l'hypomanie ainsi que la tendance habituelle à minimiser leur signification. Le thérapeute décrit les symptômes de l'hypomanie et explique au patient que les personnes souffrant de trouble BP II sont souvent incapables de reconnaître les épisodes vécus avant la thérapie car, en surface, ces épisodes peuvent paraître «normaux» ou «bons» (tout 
particulièrement en comparaison avec les épisodes dépressifs débilitants). Il est également utile de reconnaître que les hypomanies peuvent comporter des aspects positifs, tels une productivité accrue, une libido plus importante, et une plus grande créativité. La reconnaissance des aspects positifs de l'hypomanie peut faciliter ensuite une reconnaissance des aspects négatifs de ces épisodes de la part des patients, sans avoir l'impression que le thérapeute les oblige à répudier les côtés positifs de l'hypomanie valorisés par eux. "Second regard» consiste à revenir sur des hypomanies précédentes dans une phase ultérieure de la thérapie, lorsque le patient a acquis une meilleure compréhension de la maladie et de son traitement.

La reconnaissance de la nature destructrice d'hypomanies antérieures se manifesta au cours de la douzième séance de PTIRS lorsque Tanguy eut développé une meilleure compréhension de sa maladie. À cette phase du traitement, Tanguy commençait à ressentir quelques symptômes hypomaniaques et le thérapeute l'invita à revenir sur des épisodes d'hypomanie antérieures; une comparaison entre le vécu actuel et antérieur fut alors possible. Lorsque Tanguy "revisita» l'épisode hypomaniaque précédent, il se rendit compte qu'il avait minimisé dans le passé la nature dangereuse et impulsive de son comportement. Il fut capable de distinguer l'hypomanie antérieure de l'état symptomatique actuel, d'un degré beaucoup plus faible. Il admit également qu'il avait apprécié cette période de sa vie, i.e. le fait qu'il se sentait très habile socialement (ce qui n'était pas le cas dans son état habituel) et il avait le sentiment d'être attirant pour les femmes (un autre domaine de sa vie où il éprouvait des difficultés). Avec l'aide du thérapeute, il fut également capable de reconnaître les côtés "bons et mauvais" des épisodes antérieurs et de se rendre compte qu'il avait mis en péril sa propre sécurité et celle des autres par son imprudence. Il réalisa aussi que l'épisode actuel se distinguait en intensité de l'épisode antérieur et n'était pas relié à un comportement à risque. Il fut capable de relier la sévérité amoindrie de ses symptômes actuels de l'humeur à l'amélioration de la régularité de son rythme social, qui était totalement hors contrôle lorsqu'il fut arrêté. Cette compréhension encouragea Tanguy à poursuivre le travail visant l'amélioration de la régularité de ses rythmes sociaux, un processus vis-à-vis lequel il éprouvait de l'aversion au début du traitement.

Lorsqu'un patient éprouve des symptômes hypomaniaques au cours du traitement, comme dans le cas de Tanguy (que ce soit dans le contexte d'un état mixte ou d'hypomanie pure), le thérapeute devrait en profiter pour les comparer avec ceux d'hypomanies antérieures et en 
décrire les différences. Ce processus itératif aide le patient à mieux comprendre la nature des hypomanies et à évaluer de façon plus précise l'impact des épisodes sur sa vie interpersonnelle

\section{Le patient éprouve des symptômes mixtes et a des difficultés à identifier des épisodes distincts de maladie, et encore davantage, à déterminer les déclencheurs de ces épisodes.}

Lorsque les thérapeutes en PTIRS élaborent l'histoire de cas, la première phase du traitement requiert le recueil et l'établissement, avec le patient, d'un graphique de l'histoire longitudinale de la maladie qui permet d'identifier de façon précise des épisodes symptomatiques antérieurs et leurs déclencheurs. Cependant, les patients souffrant de trouble BP II éprouvent souvent des difficultés à identifier des épisodes distincts, tout particulièrement lorsque leurs épisodes sont caractérisés par un mélange de symptômes dépressifs et hypomaniaques. Parfois il est possible de distinguer les épisodes mais parfois patient et thérapeute doivent conclure que l'amalgame des symptômes est impossible à désintriquer. Néanmoins, il vaut toujours la peine de parcourir avec soin les épisodes antérieurs, en accordant une attention particulière à des déclencheurs potentiels de symptômes de l'humeur ou d'exacerbation de l'humeur.

Chantal, une femme célibataire de 43 ans, avait présenté une période de dépression dans la vingtaine qui eut pour conséquence l'abandon de ses cours universitaires, un épisode d'hypomanie dans la trentaine qui l'amena à quitter son emploi de secrétaire et à informer sa famille de son intention de déménager au Costa Rica pour y tenir une ferme biologique, et un épisode de dépression qui découla de la prise de conscience que le projet d'une ferme biologique n'était pas réaliste, et qu'elle se retrouvait sans emploi et en rupture avec les membres de sa famille. Cependant, lors de la description d'épisodes récents, elle répondit: "je me sens très mal, je me suis sentie ainsi au cours des derniers cinq ans». Elle décrivit des sensations d'agitation chronique accompagnées de pleurs, de sentiments de désespoir et d'accélération des pensées. Le contenu de ses idées accélérées était centré sur des peurs quasi-paranoüdes concernant ses voisins qu'elle croyait en colère contre elle parce qu'elle les avait accusés de consommer trop d'énergie et de ne pas avoir adhéré aux politiques de recyclage de la région. Chantal s'affichait comme "protectrice de l'environnement» ayant la conviction que la conservation commence chez soi. Elle avait pris l'habitude de faire des sondages sur les habitudes de conservation dans son voisinage (sans y être invitée) et de recommander des stratégies 
visant à améliorer la conservation de l'énergie domestique et à diminuer les déchets non recyclables. Elle eut l'impression que plusieurs de ses voisins la provoquaient en jetant des déchets dans sa cour et en volant son courrier afin de la forcer à déménager. Elle était incapable de voir un lien entre les interactions avec ses voisins et son état émotionnel; elle se sentait constamment en état d'alerte, et reconnut que cet état pouvait être justifié ou non. Afin de clarifier ses variations d'humeur, le thérapeute introduisit la MRS et demanda à Chantal de la remplir au cours de la semaine à venir. Elle expliqua à Chantal qu'elle devait évaluer le niveau d'énergie et celui de l'humeur séparément. La semaine suivante, il fut évident pour Chantal et pour le thérapeute que le niveau de l'humeur avait été stable à-2 (modérément déprimé), mais que le niveau d'énergie avait varié de bas $(-1$ à -3$)$ pour la première partie de la semaine à élevé (+2) au cours de la deuxième partie de la semaine. Chantal décrivit un sentiment de mal-être global pire dans la deuxième partie de la semaine, car elle avait ressenti de la "mauvaise énergie et non pas de la bonne énergie» (par exemple, inquiétudes, agitation, insomnie). Le thérapeute constata sur la MRS que l'augmentation de l'agitation (état "énergisé») s'était manifestée lorsque son heure de réveil avait changé de 7 heures à 10 heures. Chantal expliqua qu'elle avait été absente de la maison pendant la première partie de la semaine, travaillant dans une coopérative d'aliments biologiques. Dans la deuxième partie de la semaine, Chantal n'avait pas eu d'engagements particuliers, et elle avait été occupée à surveiller ses voisins afin de découvrir des indices de leurs comportements malveillants. Le thérapeute fut alors en mesure de suggérer que rester à la maison toute la journée avait contribué à l'augmentation de l'agitation et à une détérioration générale de son humeur. De plus, Chantal devint capable de percevoir une variation notable de son humeur, qu'elle croyait constante. Même si cette variation ne constituait pas des épisodes distincts, elle fournit au thérapeute et à Chantal un outil de départ pour examiner la relation entre humeur, énergie, rythmes sociaux et contacts sociaux.

De cette façon, le thérapeute met à la fois en relief le fait que, pour le patient, son humeur ne semble pas varier au cours des épisodes symptomatiques, mais que par ailleurs il est possible de voir, même dans les états mixtes, des variations d'intensité et de qualité dans la symptomatologie qui sont reliées à des circonstances extérieures. Le MRS fournit un moyen pour suivre de façon systématique humeur, énergie et rythmes sociaux quotidiens, ce qui permet au patient et au thérapeute de découvrir des modèles de fluctuations. Ceci donne alors au thérapeute des arguments pour inviter le patient à effectuer des changements dans 
ses rythmes sociaux et ainsi améliorer la stabilité de son énergie et de son humeur

Les patterns détectés avec l'aide de la MRS permirent au thérapeute de constater que Chantal était moins agitée les jours où elle travaillait à la coop de nourriture et demeurait loin de son voisinage. Parce que Chantal était fermement convaincue que ses interactions avec ses voisins étaient appropriées, le thérapeute se centra initialement sur une réduction du temps passé à la maison au lieu de travailler le conflit avec les voisins. Le but identifié fut la participation de la patiente à au moins une activité quotidienne à l'extérieur du voisinage. Le thérapeute expliqua que ce changement visait à l'aider à atténuer l'agitation dysphorique associée au fait de rester à la maison, et, éventuellement, à améliorer son humeur.

\section{Le patient est émotionnellement instable et changeant. Il est difficile de déterminer si le patient réagit fortement à cause de son état affectif sous-jacent ou si cet état affectif dysphorique est provoqué par sa réactivité émotionnelle.}

Dans un premier temps, le thérapeute aide le patient à reconnaître que les variations de son humeur sont caractéristiques du trouble bipolaire, et qu'il est particulièrement vulnérable aux expériences interpersonnelles qui peuvent provoquer une augmentation ou une diminution marquée de son état affectif. Le patient est encouragé à discuter et à identifier de telles expériences, passées ou actuelles. Le thérapeute et le patient commencent alors à explorer des stratégies alternatives pour gérer ces expériences tout en évitant que l'humeur du patient s'emballe dans une direction ou une autre. Le thérapeute peut suggérer différentes stratégies, comme des activités distrayantes (par exemple, regarder la télévision), ou apaisantes (par exemple, prendre un bain), une activation comportementale (par exemple, aller marcher), ou rechercher du soutien (par exemple, appeler un ami). Bien que des approches similaires aient été proposées pour le traitement du trouble de personnalité borderline, dans le cas d'un trouble BP II il importe que patient et thérapeute tiennent compte de l'humeur du patient avant d'initier quelque stratégie que ce soit. En d'autres mots, nous ne recommandons pas d'utiliser le recueillement ou la méditation (qui ralentit la pensée et l'énergie) lorsque le patient est déprimé et manque d'énergie. En général, l'approche consiste à encourager les patients à sélectionner des activités stimulantes lorsque leur énergie est basse, et à éviter de telles activités lors d'une inflation de l'humeur et/ou d'une élévation du niveau d'énergie. 
Jean-François, un homme célibataire de 24 ans, sans emploi, fut récemment délaissé par Angie, sa copine depuis 2 ans. Jean-François nota que son humeur avait varié d'extrêmement basse à très élevée au cours d'une même journée. Le thérapeute explora le contexte de ces changements et apprit que l'humeur de Jean-François s'était affaissée d'un coup, lorsqu'un ami lui envoya une messagerie texte lui apprenant qu'Angie avait une nouvelle relation amoureuse avec un ami commun. Jean-François ressentit d'abord de la colère et frappa les murs de son appartement. Ensuite il s'effondra sur son lit, abattu, et il dormit presque toute la journée. Par la suite, il se laissa convaincre de se joindre à des amis pour aller jouer au poker. Il but plusieurs bières afin de «se détendre suffisamment» pour pouvoir quitter la maison. Quand il gagna 135 \$ au jeu de poker, son humeur s'éleva et il passa le reste de la nuit avec des amis. Jean-François et le thérapeute identifièrent ensemble qu'il s'agissait là d'un cercle vicieux de comportements et d'humeur entraînant à la fois consommation d'alcool et perturbation des horaires de sommeil. Le thérapeute s'informa auprès de JeanFrançois de ce qui, en rétrospective, aurait pu l'aider à moduler sa réaction à la nouvelle concernant son ex-copine. Il mentionna que s'il avait écouté de la musique forte et ensuite était allé courir, il aurait pu éviter ses comportements négatifs (frapper le mur) et atténuer son état de dysphorie. Il reconnut aussi que s'il n'était pas resté au lit toute la journée, son sommeil n'aurait pas été perturbé et il aurait probablement ressenti un besoin moindre de boire avant de quitter la maison. Il fut aussi du même avis que le thérapeute que le jeu de poker ne fut probablement pas un bon choix d'activité, étant donné ses fluctuations d'humeur récentes. Des expériences passées lui avaient montré que le poker tendait à le surstimuler, et qu'il courait le risque de provoquer une nouvelle exacerbation de son humeur en participant à un jeu de poker, tout particulièrement après avoir bu quelques bières. Le thérapeute suggéra de faire une liste d'activités non stimulantes à entreprendre dans l'avenir s'il devait se sentir agité ou en colère, convenant qu'il pourrait perdre contrôle sur son humeur ou son comportement lors d'une surstimulation. Ils identifièrent aussi une liste d'activités stimulantes et constructives (aller courir, au lieu de jouer au poker) à utiliser s'il lui arrivait de se sentir triste et sans énergie.

Le patient est grandiose, se surestime, et peut engendrer de la colère chez les gens de l'entourage, pouvant mener à des ruptures dans les relations interpersonnelles et à des difficultés au travail.

Beaucoup de patients souffrant de trouble BP II sont très performants et ont réussi, malgré leur maladie (ou à cause de leur maladie) à 
atteindre des niveaux élevés de succès (Gartner, 2005). Parfois, cependant, l'attitude brusque et exigeante qui est garante de succès dans les salles de réunion cause des fossés interpersonnels et détruit des relations. Alors que l'hypomanie peut être acceptable au travail, les patients peuvent devoir atténuer leur attitude arrogante ou ne pas l'utiliser dans d'autres environnements. Le thérapeute aide le patient en identifiant ces comportements, en expliquant qu'ils font partie du trouble BP II, et en aidant le patient à adopter des stratégies qui lui permettent de moduler ses réactions aux autres.

Arthur est un homme marié de 64 ans, un directeur d'entreprise connu autant pour sa philanthropie flamboyante que pour ses tirades inappropriées lors de réunions d'affaires. La plupart du temps, ses collègues ignoraient ses éclats et un groupe de collègues loyaux avaient établi des stratégies pour "limiter les dégâts» suite aux diatribes d'Arthur. Durant de longues années, Arthur ne fut ni conscient ni dérangé par son comportement. Cependant, un an avant le début de son traitement, la compagnie d'Arthur fut vendue à une autre institution, et l'exécutif de la nouvelle compagnie fut moins tolérant aux idiosyncrasies d'Arthur. Il reçut plusieurs avertissements concernant son comportement et fut sur le point d'être congédié. La réaction du nouvel exécutif rendit Arthur perplexe. Il était conscient que ses connaissances et ses habiletés étaient un atout pour la compagnie, et il croyait que l'exécutif devrait l'apprécier et le récompenser plutôt que le réprimander. Il envisagea d'écrire une lettre au directeur général pour le lui dire, mais décida de l'apporter d'abord à son thérapeute pour en discuter. Son thérapeute invita Arthur à envisager la lettre du point de vue du directeur. Il mentionna que le directeur pourrait considérer la lettre comme une forme d'insubordination et comme une attaque personnelle. Arthur fut capable de reconnaître intellectuellement la possibilité d'un tel résultat. Par ailleurs, il se sentait grandement sousestimé et traité de façon tout à fait injuste. Le thérapeute expliqua que de tels sentiments pouvaient être liés à ses symptômes affectifs, l'amenant à avoir une confiance excessive en lui, dans des circonstances où d'autres seraient moins sûrs d'eux-mêmes. Ils discutèrent du fait que la constellation de ces symptômes et de son style personnel avaient pu être adéquate dans le passé, mais qu'elle l'était probablement moins dans le contexte de travail actuel. Le thérapeute utilisa des techniques PTI pour aider le patient à comprendre cette nouvelle situation comme une transition de rôles et pour l'aider à s'adapter au nouvel environnement. Après une série de jeux de rôles avec le thérapeute, visant à aider Arthur à comprendre de quelle manière ses réactions pourraient menacer ou mettre en colère des collègues, le thérapeute suggéra qu'Arthur 
compte simplement jusqu'à dix avant de dire quoi que ce soit dans des réunions importantes afin de s'accorder du temps pour "penser» avant de parler. Le thérapeute ne remit pas en question de façon directe l'idée de grande compétence et d'importance d'Arthur, mais il proposa plutôt une approche pragmatique permettant de trouver de nouvelles façons de communiquer qui seraient mieux adaptées au nouvel environnement de travail que ses anciennes stratégies.

\section{Les patients ne sont pas capables de se souvenir de la dépression lorsqu'ils sont euthymiques ou hypomaniaques et vice versa.}

Certaines personnes avec trouble BP II éprouvent une sorte «d'amnésie» pour l'humeur opposée lorsque leur polarité symptomatique change. Il est courant que les patients déprimés n'arrivent pas à se souvenir des périodes d'euthymie ou à penser qu'ils ne retrouveront jamais cet état de bien-être; à l'inverse, les patients BP I se rappellent habituellement leur état maniaque même lorsqu'ils sont déprimés. La plupart des patients avec trouble BP II, par contre, peuvent être incapables de se souvenir des hypomanies jusqu'à ce qu'ils se sentent mieux; ceci pourrait être dû au fait que ces épisodes ne sont pas aussi spectaculaires que les épisodes maniaques des BP I. Parfois, même des patients euthymiques vont éviter de discuter des périodes hypomaniaques, soit parce qu'ils ne reconnaissent pas ces périodes comme pathologiques, soit qu'ils ne se souviennent réellement pas de ces événements.

Encore plus étrange est le fait que certains patients avec trouble BP II «oublient» la dépression lorsqu'ils se sentent bien. Ils disqualifient cette période de leur vie comme ne leur ressemblant pas et se décrivent plutôt comme des personnes dynamiques. Bien que cette attitude puisse être aidante d'une certaine façon (par exemple, permettre aux patients de retrouver rapidement des niveaux de fonctionnement antérieurs sans s'attarder sur le passé récent), elle peut être défavorable à d'autres égards, car les patients peuvent perdre la motivation à continuer des activités aidantes lorsqu'ils ont l'impression que le danger de la dépression est écarté. Le thérapeute joue le rôle d'aide-mémoire pour les patients, leur rappelant des événements passés et les humeurs qui y étaient reliées. Il peut être avantageux pour certains patients de tenir un journal afin de se rappeler les expériences de dépression, même quand ces dernières sont terminées. Pour d'autres, l'utilisation d'exercices d'induction de l'humeur pendant la séance peut être préférable, ceci impliquant que le patient «reconstruit» les événements et les émotions présents lors d'un épisode dépressif récent afin d'intégrer ces expériences disparates. 
Sophia, une femme mariée de 38 ans, fut traitée pour un épisode prolongé de dépression, mais par la suite, elle annonça au thérapeute qu'elle n'avait plus besoin de traitement car elle ne pensait pas avoir un trouble bipolaire. Elle affirma que ses épisodes d'humeur avaient été "situationnels» et que le problème étant résolu, elle ne courait plus de risque d'avoir une dépression. Elle se sentait en pleine forme et projetait de mettre sur pied une nouvelle entreprise; elle craignait que la thérapie ne puisse nuire à sa capacité de se centrer sur ce nouveau projet. Le thérapeute ne manifesta pas immédiatement son désaccord avec Sophia, mais explora avec tact la compréhension qu'avait Sophia de la maladie bipolaire ainsi que ses idées justifiant que ce diagnostic ne s'appliquait pas à elle. Au cours de la discussion, le thérapeute utilisa l'humour et posa des questions additionnelles afin d'attirer l'attention sur les faiblesses dans l'argumentation de Sophia. Le thérapeute lui rappela qu'elle avait manqué des journées de travail à cause de la dépression, et que sa famille avait craint un éventuel geste suicidaire de sa part. Sophia commença à admettre qu'il lui était trop pénible de penser aux dépressions passées, et qu'elle avait toujours eu comme consigne d'aller de l'avant et de ne pas revenir sur le passé. Le thérapeute reconnut que cette stratégie l'avait aidée dans le passé, la rendant capable de faire face à une enfance traumatique et à tolérer une série de déceptions interpersonnelles au cours des ans. Par la suite, le thérapeute suggéra que Sophia avait atteint une étape de sa vie dans laquelle il pourrait être avantageux de réconcilier ces deux aspects de ses expériences de vie, afin de maintenir, lorsqu'elle se sent bien, les changements faits lors de périodes dépressives et ainsi se prémunir contre des épisodes dépressifs subséquents. Le thérapeute suggéra aussi à Sophia de considérer l'impact potentiel de trop s'investir dans un nouveau projet d'affaire. Le thérapeute rappela aussi à Sophia que des circonstances similaires avaient précédé son épisode de dépression le plus récent (Sophia était devenue déprimée dans le contexte d'avoir à se débattre pour concilier les demandes suivantes: la prise en charge d'un enfant ayant des problèmes comportementaux, prendre soin de son père malade en phase terminale, et aider son mari dans son entreprise d'aménagement paysager). Peu à peu Sophia reconnut qu'en effet, elle courait le risque de développer une autre dépression et qu'il valait la peine de poursuivre la thérapie pour l'aider à identifier et à appliquer des stratégies l'aidant à maintenir son état de bien-être. 


\section{Des patients tentent de régulariser leurs humeurs par l'abus de drogues, y compris alcool et marijuana.}

Le trouble bipolaire et les troubles d'abus de substances apparaissent souvent de façon concurrente. Tel que mentionné plus haut, les symptômes affectifs précèdent l'abus de drogues (Angst et al., 2006), ce qui suggère que les patients utilisent l'alcool et les drogues pour s' «auto-traiter». Il est utile d'expliquer ce phénomène aux patients et de suggérer que d'autres méthodes peuvent être utilisées (psychothérapie et prise de médicaments d'ordonnance) pour les aider à contrôler leurs humeurs.

Alain, un homme célibataire de 29 ans, travaillait dans la construction. Son emploi l'obligeait à commencer le travail à 6 heures. Son cycle de sommeil, tout comme celui de beaucoup de personnes avec un trouble bipolaire, présentait un délai de phase : en d'autres mots, il était naturellement un oiseau de nuit qui se couche à 2 heures du matin et se réveille à 10 heures. Il était très difficile pour lui de se réveiller à 5 heures afin d'être au travail à l'heure, mais il disait avoir besoin du revenu de ce travail. Pour pouvoir se mettre au travail, il avalait plusieurs tasses de café entre 5 et 10 heures. Il remarqua qu'il devenait très irritable plus tard dans la journée, et souvent il fumait «un joint ou deux» le soir quand il arrivait à la maison afin de «se calmer». La marijuana était agréable sur le coup, mais contribuait à une "sensation d'être à plat» qui exacerbait la fatigue matinale. Lors de sa journée de congé, il dormait jusqu'à 11 heures et évitait la caféine. Par contre, les soirs, avant de se coucher, il ressentait toujours le besoin de marijuana. Le thérapeute d'Alain fit mention d'une "déconnexion» entre sa cédule de vie et ses rythmes biologiques. À titre d'illustration, il parla de tenter d'insérer une cheville carrée dans un trou rond. Alain affirma ne pas avoir d'autres possibilités de travail et demanda, irrité: "qu'attendezvous donc de moi? ». Le thérapeute énonça l'importance de la régularité des rythmes de vie avec Alain et lui suggéra de se lever à 5 heures tous les jours, y compris les jours de congé, afin de maintenir son organisme enligné sur les mêmes cycles circadiens tous les jours de la semaine. Cette stratégie devrait, de l'avis du thérapeute, permettre à Alain de se sentir moins abattu le matin, et de lui permettre de fonctionner sans l'aide d'une aussi grande quantité de café. Alain protesta au début, disant qu'il avait hâte aux weekends pour enfin pouvoir dormir tard. Alain et le thérapeute se mirent d'accord sur un compromis, à savoir un lever à 7 heures (non pas 5 heures) les jours de congé et une réévaluation de la situation lors de la séance de la semaine suivante. Alain admit qu'après avoir fait ce changement, il avait été plus 
facile pour lui de se lever pour se rendre au travail. Bien qu'il n'eût pas encore réduit sa consommation de caféine, il pensait pouvoir réduire graduellement sa dose de café au cours des semaines suivantes. Alain se rendit éventuellement compte que son irritabilité diminuait avec la réduction de la consommation de caféine.

Lorsque l'abus de drogues est moins sévère, une stabilisation de l'humeur permet souvent une diminution ou un arrêt de l'abus. Par contre, si l'abus de substance du patient est important et représente un diagnostic supplémentaire, le thérapeute peut suggérer que le patient suive un programme d'aide adapté aux doubles diagnostics, qui offre une aide spécifique pour l'abus de substance, aide distincte de celle pour les troubles de l'humeur.

Alain réduisit éventuellement la consommation de caféine, mais continua à fumer chaque soir de la marijuana. Il ne semblait pas particulièrement intéressé à diminuer son habitude de fumer car il considérait que la marijuana était la seule chose qui l'aidait à se sentir bien à la fin de la journée. Il avait aussi l'impression que cette consommation l'aidait à dormir. Le thérapeute suggéra à Alain de rencontrer un psychiatre pour discuter avec lui d'alternatives à la prise de marijuana pour l'aider à dormir. Alain suivit partiellement la recommandation, mais continua de fumer de la marijuana malgré une prescription d'un faible dose de lorazepam. Le thérapeute utilisa une stratégie visant à "limiter les dégâts » en suggérant au patient de réduire - et non pas arrêter - sa consommation afin d'explorer s'il pouvait y avoir un lien entre la quantité fumée et son humeur. De nouveau, le patient refusa de réduire sa consommation. Après plusieurs mois de PTIRS, le thérapeute référa le patient à une clinique pour doubles diagnostics, faisant part au patient de ses doutes concernant la possibilité d'atteindre une stabilité complète de son humeur sans l'arrêt de la consommation de la marijuana, mais reconnaissant que le PTIRS ne semblait pas pouvoir lui apporter l'aide nécessaire pour la composante «dépendance » de sa maladie.

\section{Conclusion}

Nos expériences suggèrent que la PTIRS est un traitement prometteur pour des personnes souffrant de trouble BP II. Cependant, parce que cette forme de maladie bipolaire est différente du trouble BP I, une attention spéciale doit être apportée aux aspects cliniques suivants:

- explication de l'importance de la régularité des rythmes sociaux

- régulation des stimulations

- difficultés dans l'identification et la description des états affectifs 
- problèmes interpersonnels liés à la grandiosité et à la prétention

- problèmes avec la régulation de l'affect

- prévalence importante d'une comorbidité avec les abus de substance

Comme dans tous les traitements basés sur la PTI, dans l'approche PTIRS patient et thérapeute doivent devenir les experts de la maladie qui représente le focus du traitement. Ceci pourrait être un aspect particulièrement important du PTIRS dans le travail avec des patients souffrants de trouble BP II vu les manifestations complexes et changeantes de ce trouble. Les thérapeutes PTIRS jouent un rôle majeur en aidant les patients à développer la capacité d'identifier et de nommer les symptômes de l'humeur, ce qui, à son tour, donne aux patients un sentiment de contrôle, alors qu'auparavant, ils avaient l'impression que rien ne pouvait expliquer ce qui leur arrivait. Les patients (de même que les thérapeutes plus novices) trouveront profit à la lecture de livres portant sur les troubles bipolaires «atténués », afin de les aider à mieux identifier cette maladie (par exemple, Fieve, 2006; Phelps, 2006).

En résumé, PTIRS est un traitement prometteur pour le trouble BP II, et on peut raisonnablement penser à l'utiliser en monothérapie pour les patients opposés à la pharmacothérapie, ce qui n'est pas le cas avec le trouble BP I, Dans la poursuite de l'évaluation de la PTIRS pour le traitement du trouble BP II, l'un des défis consistera à identifier quels patients pourront tirer profit de la PTIRS seule et lesquels auront besoin de l'ajout d'une pharmacothérapie afin d'atteindre une stabilité d'humeur optimale.

\section{Références}

Akiskal, H. S., 2002, Classification, diagnosis and boundaries of bipolar disorder: A review, in Maj, M., Akiskal, H.S., Lopez-Ibor, J.J., eds., Evidence and Experience in Psychiatry: Bipolar Disorder, John Wiley and Sons Ltd, Chichester, England 1-52.

Akiskal, H. S., Bourgeois, M. L., Angst, J., 2000, Re-evaluating the prevalence of and diagnostic composition within the broad clinical spectrum of bipolar disorders, Journal of Affective Disorders, 59, 1, S5-S30.

American Psychiatric Association, 1994, Diagnostic and Statistical Manual of Mental Disorders, 4th ed., American Psychiatric Association, Washington, DC.

ANGST, J., 1998, The emerging epidemiology of hypomania and bipolar II disorder, Journal of Affective Disorders, 50, 143-151. 
Angst, J., Gamma, A., Benazzi, F., 2003, Toward a re-definition of subthreshold bipolarity: epidemiology and proposed criteria for Bipolar-II, minor bipolar disorders and hypomania, Journal of Affective Disorders, 73, 133-146.

Angst, J., Gamma, A., Endrass, J., 2006, Is the association of alcohol use disorders with major depressive disorder a consequence of undiagnosed Bipolar-II disorder?, European Archives of Psychiatry and Clinical Neuroscience, 256, 452-457.

Barbini, B., Colombo, C., Benedetti, F., 1998, The unipolar-bipolar dichotomy and the response to sleep deprivation, Psychiatry Research, 79, 4350 .

BeNAzZI, F., 2001a, The clinical picture of bipolar II outpatient depression in private practice, Psychopathology, 34, 81-84.

BenAzZI, F., 2001b, Course and outcome of bipolar II disorder : A retrospective study, Psychiatry and Clinical Neurosciences, 55, 67-70.

BenazzI, F., 2001c, Factor analysis of the Montgomery Asberg Depression Rating Scale in 251 bipolar II and 306 unipolar depressed outpatients, Progress in Neuro-Psychopharmacology and Biological Psychiatry, 25, 1369-1376.

BenAzZI, F., 2001d, Is the minimum duration of hypomania in Bipolar II disorder 4 days? Canadian Journal of Psychiatry/Revue canadienne de psychiatrie, 46,86 .

Coryell, W., Endicott, J., WinokuR, G., 1995, Characteristics and significance of untreated major depressive disorder, American Journal of Psychiatry, 152, 1124-1129.

Coryell, W., Keller, M., Endicott, J., 1989, Bipolar II illness : course and outcome over a five-year period, Psychological Medicine, 19, 129-141.

Dunner, D. L., Gershon, E. S., Goodwin, F. K., 1976, Heritable factors in the severity of affective illness, Biological Psychiatry, 11, 31-42.

Ehlers, C. L., Frank, E., KuPfer, D. J., 1988, Social zeitgebers and biological rhythms, Archives of General Psychiatry, 45, 948-952.

Ehlers, C. L., Kupfer, D. J., Frank, E., 1993, Biological rhythms and depression: The role of zeitgebers and zeitstorers, Depression, 1, 285-293.

FIEVE, R. R., 2006, Bipolar II : Enhance your Highs, Boost your Creativity and Escape the Cycles of Recurrent Depression: The Essential Guide to Recognize and Treat the Mood Swings of this Increasingly Common Disorder, Rodale, New York. 
Frank, E., 2005, Treating Bipolar Disorder: A Clinician's Guide to Interpersonal and Social Rhythm Therapy, Guilford Press, New York.

Frank, E., Swartz, H. A., Kupfer, D. J., 2000, Interpersonal and social rhythm therapy: Managing the chaos of bipolar disorder, Biological Psychiatry, 48, 593-604.

Gartner, J. D., 2005, The Hypomanic Edge: The Link Between (a little) Craziness and (a lot) of Success in America, Simon and Schuster, New York.

Goodwin, F., Jamison, K., 1990, Manic-Depressive Illness, Oxford University Press, New York.

Judd, L. L., Akiskal, H. S., Schettler, P. J., 2003, The comparative clinical phenotype and long term longitudinal episode course of bipolar I and II : a clinical spectrum or distinct disorders? Journal of Affective Disorders, 73, 19-32.

JudD, L. L., SchetTler, P. J., AKISKAL, H. S., 2003, Long-term symptomatic status of Bipolar I versus Bipolar II disorders, International Journal of Neuropsychopharmacology, 6, 127-137.

Koukopoulos, A., 2003, Ewald Hecker's description of cyclothymia as a cyclical mood disorder: its relevance to the modern concept of Bipolar II, Journal of Affective Disorders, 73, 199-205.

Leibenluft, E., Albert, P. S., Rosenthal, N. E., 1996, Relationship between sleep and mood in patients with rapid-cycling bipolar disorder, Psychiatry Research, 63, 161-168.

Leibenluft, E., Moul, D. E., Schwartz, P. J., 1993, A clinical trial of sleep deprivation in combination with antidepressant medication, Psychiatry Research, 46, 213-227.

LeIBENLUFT, E., SuPPES, T., 1999, Treating bipolar illness : Focus on treatment algorithms and management of the sleep-wake cycle, American Journal of Psychiatry, 156, 1976-1979.

Linehan, M. M., 1993, Cognitive-Behavioral Treatment of Borderline Personality Disorder, Guilford Press, New York.

Miklowitz, D. J., Otto, M. W., Frank, E., 2007, Psychosocial treatments for bipolar depression: A 1-Year randomized trial from the Systematic Treatment Enhancement Program, Archives of General Psychiatry, 64, 4, 419-426.

Pallanti, S., Quercioli, L., Pazzagli, A., 1999, Awareness of illness and subjective experience of cognitive complaints in patients with Bipolar I and Bipolar II Disorder, American Journal of Psychiatry, 156, 1094-1096. 
PHELPS, J., 2006, Why am I still depressed ? Recognizing and Managing the Ups and Downs of Bipolar II and Soft Bipolar Disorder, McGraw-Hill, New York.

Regier, D. A., Farmer, M. E., Rae, D. S., 1993, One-month prevalence of mental disorders in the United States and sociodemographic characteristics: the Epidemiologic Catchment Area study, Acta Psychiatrica Scandinavica, 88, 35-47.

VietA, E., Gasto, C., Otero, A., 1997, Differential Features Between Bipolar I and Bipolar II Disorder, Comprehensive Psychiatry, 38, 98-101.

Wehr, T. A., SACK, D. A., Rosenthal, N. E., 1987, Sleep reduction as a final common pathway in the genesis of mania, American Journal of Psychiatry, 144, 201-204.

Weissman, M. M., Markowitz, J. C., Klerman, G. L., 2000, Comprehensive Guide to Interpersonal Psychotherapy, Basic Books, New York.

\section{ABSTRACT}

\section{IPSRT for Bipolar II Disorder: Treatment development and case examples}

Bipolar II (BP II) disorder is a common, recurrent, and disabling psychiatric illness. Individuals suffering from this disorder comprise a large segment of the outpatient mental health treatment population, and yet little is known about how best to manage it. Psychotherapy, although untested in this population, represents a potentially important treatment modality for individuals suffering from this disorder. Because BP II disorder is characterized by subsyndromal, non-psychotic, episodes of mania (hypomania), there are no clear contraindications to the use of psychotherapy as monotherapy in BP II disorder (in contrast to BP I disorder where the risk of mania makes medication the sine qua non of treatment). In addition, unlike medication, psychotherapy has the potential to help patients address the multiple psychosocial problems associated with this chronic illness. Thus, an effective psychotherapy for BP II disorder may provide an appealing alternative for patients, especially for those who prefer to avoid the risks and discomfort associated with current pharmacotherapeutic options. Interpersonal and social rhythm therapy (IPSRT), a treatment combining a behavioral approach to increasing the regularity of daily routines with interpersonal psychotherapy (IPT), has demonstrated efficacy BP I disorder when in combination with medication. The current report gives brief overviews of BP II disorder and IPSRT, describes the process of adapting IPSRT for the treatment of BP II disorder, and then presents a series of vignettes based 
on our experience using IPSRT as monotherapy for the acute treatment of BP II depression. We argue that IPSRT warrants further systematic study to formally assess its efficacy as a treatment for BP II disorder.

\section{RESUMEN}

\section{Psicoterapia interpersonal y de ritmos sociales (PTIRS) en el trastorno bipolar II: Estructura del tratamiento y ejemplos clínicos}

El trastorno bipolar II (BPII) es una patología psiquiátrica frecuente, recurrente y debilitante. Sin embargo, pocos estudios han evaluado cuáles son los mejores enfoques para el tratamiento de esta enfermedad. La psicoterapia interpersonal y de ritmos sociales (PTIRS) ha demostrado ser útil en el tratamiento del trastorno bipolar I, en asociación con la medicación. Contrariamente al trastorno BP I, el trastorno BP II se caracteriza por episodios atenuados, no psicóticos, de manía (hipomanía), de manera que no parece haber contraindicaciones para el empleo de la PTIRS en monoterapia. Este enfoque combina un enfoque comportamental que busca aumentar la regularidad de las rutinas cotidianas, con una psicoterapia interpersonal que ayuda a los pacientes a gestionar mejor los múltiples problemas psicosociales asociados a esta patología crónica. Se realiza una descripción de las concepciones teóricas subyacentes a la utilización de la PTIRS en el trastorno bipolar y una breve descripción del trastorno BP II. Varias modificaciones parecen necesarias, en nuestra investigación, para adaptar la PTIRS al tratamiento del trastorno BP II (en comparación con el trastorno BP I), en razón de las características clínicas particulares de esta población, específicamente la inestabilidad del cuadro clínico, la dificultad de localizar el tipo de episodio en curso porque los síntomas son con frecuencia mixtos (intrincación entre síntomas de activación y de depresión), e igualmente la razón de la superposición sintomática o de la comorbilidad con el trastorno de personalidad límite. Existe también una comorbilidad frecuente con el trastorno de abuso o dependencia a sustancias psicoactivas. Las viñetas tomadas de nuestra experiencia clínica intentan ilustrar diversas problemáticas comunes encontradas en la terapia de esta población y que están relacionadas con las características del trastorno BP II mencionadas anteriormente. La PTIRS parece ser una adición importante e interesante a los enfoques de tratamiento del trastorno BP II porque toma en cuenta los diversos aspectos de esta patología. Este enfoque nos parece eficaz en este estudio preliminar y somos de la opinión que se requieren estudios sistemáticos posteriores que permitirán evaluar de manera más formal su eficacia en el tratamiento del trastorno bipolar II. 


\section{RESUMO}

\section{Psicoterapia interpessoal e de ritmos sociais (TIPRS) no transtorno bipolar II: Estrutura do tratamento e exemplos clínicos}

O transtorno bipolar II (BP II) é uma patologia psiquiátrica frequiente, recorrente e debilitante. Entretanto, poucos estudos avaliaram quais eram as melhores abordagens para o tratamento desta doença. A psicoterapia interpessoal e de ritmos sociais (TIPRS) demonstrou uma utilidade no tratamento do transtorno bipolar I, associado à medicação. Ao contrário do transtorno BP I, o transtorno BP II é caracterizado por períodos atenuados, não psicóticos, de mania (hipomania), de maneira que não parece ter contra-indicações no emprego da TIPRS em monoterapia. Esta abordagem associa uma abordagem comportamental visando a aumentar a regularidade das rotinas cotidianas, com uma psicoterapia interpessoal que ajuda os pacientes a melhor gerir os múltiplos problemas psicossociais associados a esta patologia crônica. É feita uma descrição dos conceitos teóricos que subentende a utilização da TIPRS no transtorno bipolar. Uma breve descrição do transtorno BP II é feita. Várias modificações mostraram-se necessárias, em nossa experiência, para adaptar a TIPRS ao tratamento do transtorno BP II (em comparação com o transtorno BP I), por causa das características clínicas particulares desta população, principalmente com respeito à instabilidade do quadro clínico, à dificuldade de perceber o tipo de episódio em curso porque os sintomas são freqüentemente mistos (combinação entre sintomas de ativação e de depressão), e igualmente, por causa da acumulação sintomática ou da comorbidade com o transtorno de personalidade borderline. Existe também uma co-morbilidade freqüente com o transtorno do abuso ou de dependência às substâncias psicoativas. Os exemplos tirados de nossa experiência clínica tendem a ilustrar diversas problemáticas correntes encontradas na terapia desta população, e que são relacionadas às características acima mencionadas do transtorno BP II. A TIPRS parece ser um acréscimo importante e interessante às abordagens de tratamento do transtorno BP II porque ela leva em consideração diversos aspectos desta patologia; esta abordagem nos parece eficaz neste estudo preliminar, e nós acreditamos que estudos sistemáticos futuros são necessários e permitirão avaliar de maneira mais formal sua eficácia no tratamento do transtorno bipolar II. 